\title{
GROUPS GENERATED BY TWO OPERATORS EACH OF WHICH IS TRANSFORMED INTO A POWER OF ITSELF BY THE SQUARE OF THE OTHER.
}

BY PROF ESSOR G. A. MILLER.

(Read before the Chicago Section of the American Mathematical Society, January 1, 1910.)

\section{§1. Introduction.}

Two special cases of the category of groups defined by the heading of this paper have been considered; viz., when the square of each of the two generators transforms the other generator either into itself* or into its inverse. $\dagger$ It was observed that in the former of these two cases the orders of the two generators are not restricted, while in the latter each of these orders must divide 8. Each of these special cases led to a very elementary category of solvable groups. It will be proved that the more general category defined by the heading of this paper is also composed entirely of solvable groups of simple structure.

As an instance of how such generalizations may lead to very complex categories of groups we may give the theorem that every symmetric group can be generated by two operators whose squares are commutative. In fact, the symmetric group of degree $n$ is evidently generated by the following two cyclic substitutions whose squares are commutative :

$$
t_{1}=\left(x_{1} x_{2} x_{3} \cdots x_{n-1}\right), \quad t_{2}=\left(x_{1} x_{n}\right) .
$$

From the theorem that every symmetric group whose degree exceeds 8 can be generated by two substitutions of orders 2 and 3 respectively $\ddagger$ it results directly that all such groups are included in the category of groups defined by the condition that each of them can be generated by two operators which are transformed into themselves by the square and cube respectively of the other.

* Bulletin, vol. 16 (1910), p. 173.

$\ddagger$ Annals of Mathematics, vol. 9 (1907), p. 48.

$\ddagger$ Bulletis, vol. 7 (1901), p. 426. 


\section{§ 2. General Considerations.}

The conditions imposed upon the two generators $s_{1}, s_{2}$ of the group $G$, as expressed by the heading of this paper, give rise to the following equations :

$$
8_{1}^{-2} s_{2} s_{1}^{2}=s_{2}^{a}, \quad s_{2}^{-2} s_{1} s_{2}^{2}=s_{1}^{\beta} .
$$

If at least one of the two numbers $\alpha, \beta$ is even, the corresponding operator is of odd order and hence it must be generated by its square. In this case $G$ is generated by a cyclic group and an operator transforming this cyclic group into itself. As many properties of these groups are well known, we shall confine our attention, in what follows, to the consideration of cases in which both $\alpha$ and $\beta$ are odd. The group $H$ generated by $s_{1}^{2}, s_{2}^{2}$ clearly belongs to the elementary category of groups which may be generated by two operators each of which transforms the other into a power of itself.* Hence the commutator subgroup of $H$ is cyclic and the order of $H$ divides the quotient obtained by dividing the product of the orders of $s_{1}^{2}, s_{2}^{2}$ by the order of the commutator subgroup.

It is easy to see that $H$ is invariant under $G$, since $\alpha$ and $\beta$ are odd and the following equations are satisfied :

$$
s_{1}^{-1} s_{2}^{2} s_{1}=s_{2}^{2} s_{2}^{-2} s_{1}^{-1} s_{2}^{2} s_{1}=s_{2}^{2} s_{1}^{1-\beta}, \quad s_{2}^{-1} s_{1}^{2} s_{2}=s_{1}^{2} s_{2}^{1-a} .
$$

From the fact that

it results that

$$
s_{2}^{-2} s_{1}^{-2} s_{2}^{2}=s_{2}^{2 \alpha-2} s_{1}^{-2}=s_{1}^{-2 \beta}
$$

$$
s_{1}^{2(\beta-1)} s_{2}^{2(a-1)}=1 \text {. }
$$

Hence the two operators $s_{1}^{2(\beta-1)}, s_{2}^{2(\alpha-1)}$ are invariant under $G$. To find multiples of the orders of $s_{1}, s_{2}$ we may transform these invariant operators by $s_{2}^{2} s_{1}^{2}$ respectively, as follows :

$$
\begin{aligned}
& s_{2}^{-2} s_{1}^{2(\beta-1)} s_{2}^{2}=s_{1}^{2 \beta(\beta-1)}=s_{1}^{2(\beta-1)}, \quad \text { or } \quad s_{1}^{2(\beta-1)^{2}}=1 \text {; } \\
& s_{1}^{-2} s_{2}^{2(\alpha-1)} s_{1}^{2}=s_{2}^{2 \alpha(a-1)}=s_{2}^{2(a-1)}, \quad \text { or } \quad s_{2}^{2(a-1)^{2}}=1 \text {. }
\end{aligned}
$$

Hence the theorem: If two operators $s_{1}, s_{2}$ satisfy the equations $s_{1}^{-2} s_{2} s_{1}^{2}=s_{2}^{a}, s_{2}^{-2} s_{1} s_{2}^{2}=s_{1}^{\beta}$, their orders divide $2(\beta-1)^{2}$ and $2(\alpha-1)^{2}$ respectively, their squares generate a group which involves a cyclic commutator subgroup and is invariant under the

* Quar. Jour. of Math., vol. 37 (1906), p. 286. 
group generated by $s_{1}$ and $s_{2}$, and they, satisfy the equation $s_{1}^{2(\beta-1)}=s_{2}^{2(1-a)}$.

The quotient group of $G$ with respect to $H$ is dihedral, since it can be generated by two operators of order 2 . As $H$ is solvable and this quotient group is solvable, $G$ is always solvable. That is, if two operators are such that each is transformed into a power of itself by the square of the other, they generate a solvable group whose fourth derived is identity. As an instance of such a group, we may cite the symmetric group of order 24 . This is generated by any two of its operators of order 4 which do not have a common square, and each of these two operators is transformed into its inverse by the square of the other. In this case the third derived is already identity. To obtain a group whose third derived is not identity we may consider the group of order 48 generated by two operators of order 8 each of which is transformed into its inverse by the square of the other.* This group of order 48 illustrates also that the orders of $s_{1}, s_{2}$ may be actually $2(\beta-1)^{2}, 2(\alpha-1)^{2}$ respectively.

The theorem stated above implies that the numbers $\alpha, \beta$ always fix an upper limit for the orders of $s_{1}, s_{2}$ except when at least one of these numbers is unity. When both of them are unity there results the special case noted in the introduction. If only one of them $(\alpha)$ is unity the defining relations assume the form

$$
s_{1}^{-2} s_{2} s_{1}^{2}=s_{2}, \quad s_{2}^{-2} s_{1} s_{2}^{2}=s_{1}^{\beta}(\beta \neq 1) .
$$

Hence $2=2 \beta+k n, n$ being the order of $s_{1}$ and $k \neq 0$. That is, if we assume $\alpha=1, \beta \neq 1$, it results that the order of $s_{1}$ is a divisor of $2(\beta-1)$. These conditions do not fix an upper limit for the order of $s_{2}$, as may be seen from the following special case. The two operators $s_{1}, s_{2}$ may evidently be the generators of the dicyclic group of order $16, s_{2}$ generating the cyclic group of order 8 and $\beta$ being 3 . If $s_{2}$ is multiplied by an operator of arbitrary order which is commutative with each of the operators $s_{1}, s_{2}$, the product thus obtained and $s_{1}$ will again satisfy the given conditions. This proves that the order of $s_{2}$ may be an arbitrary multiple of 8 when $\alpha=1$ and $\beta=3$, but the order of $s_{1}$ must be 4 .

It was observed above that the orders of $s_{1}, s_{2}$ divide $2(\beta-1)^{2}$, $2(\alpha-1)^{2}$ respectively and that the $2(\beta-1)$ th power of the former of these operators is equal to the $2(1-\alpha)$ th power of the

\footnotetext{
*Annals of Mathematics, vol. 9 (1907), p. 51.
} 
latter. Hence it results that the order of each of these operators is a divisor of $2(\alpha-1)(\beta-1)$. That is, if two operators are such that each is transformed into a power of itself by the square of the other, the order of each of them is a divisor of twice the product of the indices of these powers diminished by unity. When $\alpha$ and $\beta$ are different this evidently furnishes a lower limit for the order of one of the operators than the one given before. Since $s_{1}^{2(\beta-1)}$ and $s_{2}^{2(a-1)}$ are invariant under $G$, it results that

$$
s_{1}^{\beta^{\alpha-1}}=s_{1}, \quad s_{2}^{a^{\beta-1}}=s_{2} .
$$

Hence the order of $s_{1}$ is a divisor of $\beta^{a-1}-1$, and that of $s_{2}$ is a divisor $\alpha^{\beta-1}-1$. These conditions will sometimes give lower limits for these orders than those given above, as may be seen from the special cases in the following section.

The preceding considerations prove that two operators which satisfy the two conditions

$$
s_{1}^{-2} s_{2} s_{1}^{2}=s_{2}^{a}, \quad s_{2}^{-2} s_{1} s_{2}^{2}=s_{1}^{\beta}
$$

have their orders limited by these conditions when, and only when, each of the numbers $\alpha, \beta$ is different from unity. When $\alpha, \beta$ satisfy this condition, $s_{1}^{2}, s_{2}^{2}$ generate an invariant subgroup whose order is a divisor of the product of their orders. For every pair of odd values for $\alpha, \beta$ there is an infinite system of groups whose fourth derived must be unity and each of these systems must include the dihedral groups. The properties of the invariant subgroup generated by $s_{1}^{2}, s_{2}^{2}$ are known, since these operators transform each other into powers, and every $G$ may be constructed by adjoining to this invariant subgroup the operator $s_{1} s_{2}$ and then adjoining to the invariant subgroup thus obtained the operator $s_{1}$. While these fundamental properties apply to every possible $G$, a number of interesting special properties apply to given values of $\alpha$ and $\beta$, as may be seen from the examples of the following section.

\section{§3. Special Cases.}

The following special cases may serve to illustrate some of the preceding theorems. Suppose that $s_{1}, s_{2}$ are any two operators which satisfy the two equations

$$
s_{1}^{-2} s_{2} s_{1}^{2}=s_{2}^{3}, \quad s_{2}^{-2} s_{1} s_{2}^{2}=s_{1}^{3} .
$$

From the general results of the preceding section it follows that $s_{1}^{4}=s_{2}^{4}, s_{1}^{8}=s_{2}^{8}=1$, and that $s_{1}, s_{2}$ have the same order when- 
ever neither of these operators is identity. When $s_{1}, s_{2}$ are commutative they generate the four-group or a subgroup of this group. This trivial case will be excluded in what follows. That is, we shall assume that $s_{1}, s_{2}$ are non-commutative. When each of these operators is of order 2 they generate a dihedral group and every dihedral group may evidently be generated by two operators satisfying the given conditions. The only two possible cases which remain to be considered are when the common order of $s_{1}, s_{2}$ is either 4 or 8 . In each of these cases the order of $s_{1} s_{2}$ is a multiple of 3 since

$$
\begin{gathered}
s_{1}^{-1} s_{2}^{-1} \cdot s_{2}^{2} \cdot s_{2} s_{1}=s_{1}^{-1} s_{2}^{2} s_{1}=s_{2}^{2} \cdot s_{2}^{-2} s_{1}^{-1} s_{2}^{2} \cdot s_{1}=s_{2}^{2} s_{1}^{-2}, \\
s_{1}^{-1} s_{2}^{-1} \cdot s_{2}^{2} s_{1}^{-2} \cdot s_{2} s_{1}=s_{1}^{-1} s_{2} s_{1}^{-2} s_{2} s_{1}=s_{1}^{-3} \cdot s_{1}^{2} s_{2} s_{1}^{-2} \cdot s_{2} s_{1}=s_{1}^{-2} s_{2}^{4}=s_{1}^{2}, \\
s_{1}^{-1} s_{2}^{-1} \cdot s_{1}^{2} \cdot s_{2} s_{1}=s_{1} \cdot s_{1}^{-2} s_{2}^{-1} s_{1}^{2} \cdot s_{2} s_{1}=s_{1} s_{2}^{-2} s_{1}=s_{2}^{-2} \cdot s_{2}^{2} s_{1} s_{2}^{-2} s_{1}=s_{2}^{2} .
\end{gathered}
$$

When the common order of $s_{1}, s_{2}$ is 4 the invariant subgroup $H$ generated by $s_{1}^{2}, s_{2}^{2}$ is the four-group. If the order of $s_{1} s_{2}$ is $3, G$ must be the symmetric group of order 24 . In fact, in this case $G$ is generated by two operators which are such that each is transformed into its inverse by the square of the other and hence its properties are known, as was observed in the introduction. The only case which remains to be considered is the one where the common order of $s_{1}, s_{2}$ is 8 . In this case $H$ is the quaternion group and the order of $G$ is a multiple of 48 . When the order of $s_{1}, s_{2}$ is $3, G$ is one of the four groups of order 48 which involve the non-twelve group of order 24 . Since $\left(s_{1} s_{2}^{2}\right)^{2}=s_{1} s_{2}^{2} s_{1} s_{2}^{2}=s_{1} s_{2}^{4} \cdot s_{2}^{-2} s_{1} s_{2}^{2}=1$, this group can be represented as a transitive substitution group on 8 letters. It is easy to verify that the following generators of this transitive substitution group satisfy the conditions imposed upon $s_{1}, s_{2}$ :

$$
s_{1}=a c f h b d e g, \quad s_{2}=\text { agdfbhce. }
$$

These generators are directly obtained from those given by Cole.*

From what precedes it is not difficult to deduce some fundamental properties of the infinite system of groups generated by two operators of order 8, each of which is transformed into its third power by the square of the other. In each of these groups $\left(s_{1} s_{2}\right)^{3}$ generates an invariant subgroup whose operators are separately invariant under half the operators of the entire

*Bulletin, vol. 2 (1893), p. 188. 
group, while they are transformed into their inverses by the remaining operators. The fourth derived of each of these groups is identity while the third derived is of order 2. The quotient group of $G$ with respect to the subgroup generated by $\left(s_{1} s_{2}\right)^{3}, s_{1}^{2}, s_{2}^{2}$, is the symmetric group of order 6 and this subgroup has the group of order 2 for its commutator subgroup. The quotient group of $G$ with respect to the invariant group generated by $\left(s_{1} s_{2}\right)^{3}$ is either the symmetric group of order 24 or the group of 48 which may be generated by the two substitutions given in the preceding paragraph. Hence the theorem: If two non-commutative operators are such that each is transformed into its third power by the square of the other, they are of the same order and this common order is 2,4 , or 8 . If this order is 2 they generate a dihedral group; if it is 4 they generate a group whose quotient group with respect to a cyclic invariant subgroup is the symmetric group of order 24 ; and if it is 8 the corresponding quotient group is either this symmetric group or a group of order 48 which can be represented as a transitive substitution group of degree 8.

If $s_{1}, s_{2}$ satisfy the two conditions

$$
s_{1}^{2-} s_{2} s_{1}^{-2}=s_{2}^{5}, \quad s_{2}^{-2} s_{1} s_{2}^{2}=s_{1}^{5},
$$

their orders divide 16 , since $5^{4}-1$ is not divisible by 32 . It also follows from the theorems of the preceding section that $s_{1}^{8} s_{2}^{8}=1$, and hence $s_{1}^{8}=s_{2}^{8}$. To verify that the common order of $s_{1}, s_{2}$ may actually be 16 we may employ the following substitutions :

$$
s_{1}=a b c d e f g h i j k l m n o p, \quad s_{2}=\text { ahcfmlojipknedgb. }
$$

When $s_{1}, s_{2}$ represent these substitutions, $H$ is of order 32 and involves a commutator subgroup of order 2 . Since

$$
s_{1} s_{2}=b f \cdot \mathrm{cg} \cdot \mathrm{em} \cdot h p \cdot j n \cdot k o
$$

the order of $G$ is 128 . In the next paragraph we shall prove that the order of $H$ is 32 and that the order of $G$ is a multiple of 128 whenever $s_{1}$ is of order 16 .

The operator $\left(s_{1} s_{2}\right)^{2}$ is invariant under half the operators of $G$ while it is transformed into its inverse by the remainder of these operators since

$$
\begin{aligned}
\left(s_{1} s_{2}\right)^{-2}=s_{2}^{-1} s_{1}^{-1} s_{2}^{-1} s_{1}^{-1} & =s_{2} \cdot s_{2}^{-2} s_{1}^{-1} s_{2}^{2} \cdot s_{2}^{-3} s_{1}^{-1} \\
& =s_{2} s_{1}^{-5} s_{2}^{-3} s_{1}^{-1}=s_{2} s_{1} \cdot s_{1}^{-6} s_{2}^{-3} s_{1}^{6} \cdot s_{1}^{-7}=\left(s_{2} s_{1}\right)^{2} .
\end{aligned}
$$


As $\left(s_{1} s_{2}\right)^{2}$ is transformed into its inverse by $s_{1}$, it is also transformed into its inverse by $s_{2}$ and hence it is transformed into itself by half the operators of $G$. To prove that the order of $H$ is exactly 32 when $s_{1}$ is of order 16 it is only necessary to observe that $s_{1}^{4}$ is not invariant under $G$, and this results from the continued equation

$$
\left(s_{1} s\right)^{-1} s_{1}^{4} s_{1} s_{2}=s_{2}^{-1} s_{1}^{4} s_{2}=s_{1}^{4} \cdot s_{1}^{-4} s_{2}^{-1} s_{1}^{4} \cdot s_{2}=s_{1}^{4} s_{2}^{8}=s_{1}^{-4} .
$$

These results give rise to the theorem : If two operators of order 16 are such that each of them is transformed into its fifth power by the square of the other, these squares generate an invariant subgroup of order 32 involving a commutator subgroup of order 2 .

It has been proved that the group generated by $\left(s_{1} s_{2}\right)^{2}$ is invariant under $G$ and that the order of the corresponding quotient group is a divisor of 128 . To prove that this invariant subgroup has at most two operators in common with the invariant subgroup $H$ it is only necessary to observe that a subgroup of order 4 in the former of these invariant subgroups can not be contained in the latter. This results directly from the facts that $s_{1} s_{2}$ is not commutative with $s_{1}^{4}$ or $s_{2}^{4}$ and that the operators of order 4 in $H$ which are not generated by $s_{1}^{4}$ or $s_{2}^{4}$ are non-commutative with $s_{1}^{2}$, while $\left(s_{1} s_{2}\right)^{2}$ is commutative with this operator. Since the commutators of $G$ are contained in the invariant subgroup generated by $s_{1}^{2}, s_{2}^{2},\left(s_{1} s_{2}\right)^{2}$, and this invariant subgroup has a commutator subgroup of order 2 , it results that the second derived of $G$ is identity. It is also evident that the quotient group of $G$ with respect to this invariant subgroup is the four-group. Hence the theorem : If each of two operators is transformed into its fifth power by the square of the other, the orders of these operators divide 16 and the second derived of the group generated by them is identity. Hence these operators may have a common order only when this order is $1,2,4,8$, or 16 , and if they are non-commutative they can have different orders only when these orders are one of the two pairs 2,$4 ; 4,8$.

As a third and final special case we consider the relations

$$
s_{1}^{-2} s_{2} s_{1}^{2}=s_{2}^{3}, \quad s_{2}^{-2} s_{1} s_{2}^{2}=s_{1}^{5},
$$

where $\alpha$ and $\beta$ are unequal. According to the theorems of the preceding section, the orders of $s_{1}, s_{2}$ divide 8 and 4 respectively. It is easy to verify by means of substitutions that the orders of $s_{1}, s_{2}$ may actually be 8 and 4 respectively. 


$$
s_{1}=a b c d e f g h \cdot i j l m n o p, \quad s_{2}=\text { aiem } \cdot \operatorname{cog} k \text {. }
$$

When $s_{1}$ is of order $8, s_{2}$ must be of order 4 since $s_{2}^{2}$ is not commutative with $s_{1}$. When $s_{1}$ is of order 4 , the order of $s_{2}$ is either 4,2 , or 1 , as may be readily seen from the following substitutions :

$$
s_{1}=a e c g, \quad s_{2}=a b c d \cdot e f g h ; \quad s_{1}=a b c d, \quad s_{2}=a c .
$$

Finally, when $s_{1}$ is of order 2 , the order of $s_{2}$ is evidently 2 or 1 . Hence the theorem : If two non-commutative operators satisfy the relations $s_{1}^{-2} s_{2} s_{1}^{2}=s_{2}^{3}, \quad s_{2}^{-2} s_{1} s_{2}^{2}=s_{1}^{5}$, their orders are one of the following pairs of numbers: 8,$4 ; 4,4 ; 4,2 ; 2,2$.

When $s_{1}$ is of order $8, H$ is abelian and of order 8 . From the following equations it results that $s_{1}^{2}$ is transformed into its inverse by $\left(s_{1} s_{2}\right)^{2}$ :

$$
\begin{gathered}
\left(s_{1} s_{2}\right)^{-1} s_{1}^{2} s_{1} s_{2}=s_{2}^{-1} s_{1}^{2} s_{2}=s_{1}^{2} \cdot s_{1}^{-2} s_{2}^{-1} s_{1}^{2} \cdot s_{2}=s_{1}^{2} s^{2} \\
\left(s_{1} s_{2}\right)^{-2} s_{1}^{2}\left(s_{1} s_{2}\right)^{2}=s_{2}^{-1} s_{1} s_{2}^{2} s_{1} s_{2}=s_{2} \cdot s_{2}^{-2} s_{1} s_{2}^{2} \cdot s_{1} s_{2}=s_{2} s_{1}^{-2} s_{2}=s_{1}^{-2} .
\end{gathered}
$$

Hence the order of $G$ is a multiple of $8 \cdot 4 \cdot 2=64$ whenever $s_{1}$ is of order 8 . That the order of $G$ may be exactly 64 results directly from the given substitutions, as they generate an imprimitive group of degree 16 and order 64 . From the properties of the dihedral group it results that $s_{1}, s_{2}$ may be so selected that the order of $G$ is an arbitrary multiple of 64 and that the third derived of each one of these groups is identity. The categories of groups which result when the orders of $s_{1}, s_{2}$ have the other possible sets of values are still more elementary and their fundamental properties are easily derived from the general theorems of the preceding section.

\section{THE SOLUTION OF AN INTEGRAI EQUATION OCCURRING IN THE THEORY OF RADIATION.}

BY PROFESSOR W. H. JACKSON.

(Read before the American Mathematical Society, December 30, 1909.)

Professor Arthur Schuster* has discussed the propagation of heat by radiation when the isothermal surfaces are

\footnotetext{
* "The influence of radiation on the transmission of heat." Phil. Magazine, Feb., 1903.
} 